\title{
Aceitação da utilização de metodologias ativas nos estágios no SUS por discentes da graduação e pós-graduação em Odontologia
}

Wellington Mendes Carvalho*, Patrícia Tiemi Cawahisa*, Paula Cabrini Scheibel*, Juliana Nunes Botelho**, Raquel Sano Suga Terada***, Najara Barbosa Rocha***, Luiz Fernando Lolli***, Mitsue Fujimaki***

* Mestre em Odontologia Integrada - Universidade Estadual de Maringá, PR

** Doutora em Odontologia - Universidade Estadual de Campinas, SP

*** Professores do Departamento de Odontologia Universidade Estadual de Maringá, PR

\section{RESUMO}

O objetivo do estudo foi avaliar a aceitação da utilização de metodologias ativas de ensinoaprendizagem nos estágios no SUS (Sistema Único de Saúde) por discentes da graduação e pós-graduação em Odontologia. Foi realizada uma pesquisa de opinião, com análise de dados de forma quanti-qualitativa. Para tanto, 30 estudantes de graduação e 10 de pósgraduação responderam a um questionário semiestruturado contendo perguntas relativas às suas experiências com esta metodologia. Os dados revelaram que $67 \%$ dos estudantes de graduação e $70 \%$ da pós-graduação nunca haviam entrado em contato com metodologias ativas de ensino-aprendizagem. Houve satisfação com a metodologia adotada, aprovada pela totalidade dos alunos. As metodologias ativas mostraram-se como alternativas didático-pedagógicas viáveis, com aceitação entre os estudantes de graduação e pós-graduação, estando em consonância com as Diretrizes Curriculares Nacionais para os Cursos de Odontologia no Brasil.
Descritores: Odontologia. Aprendizagem Baseada em Problemas, Educação Superior.

\section{INTRODUÇÃO}

A exigência de um profissional com competências e habilidades, bem como flexibilidade para continuar aprendendo ao longo da vida profissional, é cada vez maior diante de demandas sociais tão complexas, pois requer um crescimento acelerado do volume de conhecimento ${ }^{1}$. Exige-se que o profissional de saúde tenha uma formação sólida que contemple tanto o conhecimento em sua área de atuação, como as habilidades e atitudes $^{2}$. Há uma necessidade de mudanças na graduação do cirurgião-dentista para que seja possível formar os profissionais de forma generalista, humanista, crítica e reflexiva ${ }^{3-5}$, ficando cada vez mais evidente a fragilidade do modelo de ensino conhecido como "tradicional" ou de transmissão, centrado na figura do professor que detém e transmite conhecimento, que cria uma distância entre 
teoria e prática e consequentemente, um desconhecimento da realidade ${ }^{6,7}$.

As tendências da área da Educação apontam para a utilização de metodologias ativas de ensino, que têm no aluno o centro do processo ensino-aprendizagem, sendo este o protagonista do seu próprio processo de formação. Duas das principais metodologias ativas utilizadas nos cursos da área de saúde no mundo são a Aprendizagem Baseada em Problemas (ABP) ou Problem-based Learning (PBL), onde todo o currículo é planejado de forma a utilizar metodologias ativas, e a Metodologia da Problematização, que pode também ser adotada como norteadora de todo o currículo ou em apenas uma disciplina ou curso $^{8}$. Nestas duas propostas, a utilização de problemas reais (problematização) ou a criação de problemas por uma equipe de especialistas (ABP), são o norte para o processo ensino-aprendizagem. No Brasil, até o momento, nenhum curso de Odontologia adotou a ABP como estrutura curricular, mas cursos em outros países tem sido inspiradores para ultrapassar a abordagem tradicional ${ }^{8}$. Junto com a integração ensino-serviço, a utilização de metodologias ativas de ensino-aprendizagem é apontada como estratégia para a formação voltada para o mundo do trabalho e para as necessidades da população, visando o aprendizado significativo, ou seja, quando o aluno adquire novos conhecimentos mediante seu próprio esforço, ligando conceitos ou proposições relevantes preexistentes ${ }^{5,8,9}$.

Alguns exemplos de utilização da problematização nos cursos de Odontologia podem ser citados como a disciplina de Fisiologia na FOP-UNICAMP ${ }^{10}$, Disciplina de Periodontia na $\mathrm{UEPG}^{11}$, disciplinas isoladas do curso de Odontologia da UNIFOR $^{12}$ e da UNIPLAC $^{13}$, disciplina de
Oclusão na FO-UFG ${ }^{14}$ e na disciplina de Estomatologia da UFES ${ }^{15,16}$.

É válido citar, além da PBL e a problematização, outros tipos de metodologias ativas que podem ser utilizadas no processo ensino-aprendizagem: portfólio, internet, dramatização, discussão, seminários, estudos de casos, entre outros ${ }^{9,17-19}$

Em 1992, o Curso de Odontologia da Universidade Estadual de Maringá (UEM) implementou mudanças no projeto pedagógico baseadas em um currículo integrado, já contando com uma carga horária de estágio totalizando $20 \%$ do total do curso. Estas mudanças foram consideradas inovadoras e se encontravam em consonância com as Diretrizes Curriculares Nacionais (DCN) para os cursos de graduação em Odontologia $^{20}$, que só entraram em vigor a partir de 2002. No ano de $2005^{21}$, o curso teve seu projeto aprovado pelo Programa Nacional de Reorientação da Formação Profissional em Saúde (Pró-Saúde) e entre as ações previstas, pode-se destacar a capacitação para uso de novas metodologias de ensino-aprendizagem. Assim, a implementação de metodologias ativas se iniciou no ano de 2006, nas atividades do $5^{\circ}$ ano da graduação na disciplina Estágio Supervisionado IV, referentes à prática dentro da Estratégia Saúde da Família. A principal metodologia utilizada na disciplina foi a problematização. Além disso, a preocupação em formar docentes aptos a ensinar utilizando essa metodologia surgiu com a criação do Programa de Mestrado em Odontologia Integrada da UEM, em 2008, onde a primeira turma de mestrandos puderam vivenciar as metodologias ativas em dois créditos do curso: Introdução à Saúde Coletiva e Currículo Integrado e Integração Multiprofissional. 
Apesar dos relatos na literatura sobre a utilização de metodologias ativas de ensinoaprendizagem ${ }^{10-16}$ na área da Odontologia até o momento, poucos trabalhos têm demonstrado a aplicação destas na integração do serviço de saúde ao ensino da Odontologia. Desta maneira, o objetivo do estudo foi avaliar a aceitação de discentes de graduação e pósgraduação stricto sensu do Curso de Odontologia e do Programa de PósGraduação em Odontologia Integrada da UEM sobre a utilização de metodologias ativas de ensino-aprendizagem.

\section{MÉTODOS}

Este trabalho foi aprovado pelo Comitê de Ética em Pesquisa da UEM sob o protocolo CAAE n. 0409.0.093.000-08, de 13 de fevereiro de 2009.

Tratou-se de um relato de uma experiência realizada em 2009, na área de Saúde Coletiva do Curso de Odontologia da UEM, com análise dos dados de forma quantiqualitativa. Participaram deste estudo 30 acadêmicos do último ano da graduação em Odontologia que participavam da disciplina de Estágio Curricular Supervisionado em Saúde Coletiva IV e todos os acadêmicos da pós-graduação Strictu Sensu em Odontologia Integrada da UEM.

Os critérios de inclusão da amostra incluíram os indivíduos que consentiram em participar da pesquisa respondendo a um questionário semiestruturado, contendo questões abertas e fechadas, que abordavam impressões e opiniões sobre a metodologia de ensino-aprendizagem empregada durante os encontros. O questionário foi elaborado a partir de uma revisão da literatura prévia do tema e de avaliações rotineiras anuais dos estágios e disciplinas da pós-graduação.

Os alunos da graduação foram divididos em grupos de no máximo sete pessoas, com dois tutores para cada grupo. Nesta atividade, foram abordados o histórico das políticas públicas de saúde no Brasil, implantação do SUS e suas principais diretrizes. O estudo se dividiu em três etapas:

1. Identificação do problema: por meio de um texto previamente elaborado, os alunos de graduação, com a ajuda dos tutores, foram expostos a um problema onde conhecimentos sobre as políticas de saúde e sobre o SUS eram a temática. Assim, os alunos expressaram seu conhecimento prévio sobre o tema e formularam perguntas para resolverem suas dúvidas.

2. Teorização do assunto: no segundo encontro, os alunos trouxeram suas pesquisas sobre as perguntas formuladas e houve uma nova discussão, na qual todo o conteúdo teórico pesquisado pelos graduandos foi exposto, com complementações didáticas dos tutores.

3. Fechamento do estudo: após as discussões, o grupo elaborou uma síntese, onde todos os conhecimentos adquiridos foram explicitados. Essa síntese foi exposta em uma reunião final, com todos os grupos juntos.

Essa abordagem metodológica foi empregada pela primeira vez em nível de graduação no curso.

\section{RESULTADOS}

Os resultados às perguntas fechadas estão expressos em porcentagens na tabela 1 . Verifica-se que todos os alunos que participaram da pesquisa, tanto em nível de graduação quanto de pós-graduação, responderam que a utilização de metodologias ativas durante a graduação é uma experiência válida. Mais da metade dos alunos não havia participado até então de alguma atividade de 
ensino que empregava metodologia ativa. A respondeu que se sente muito à vontade para maioria deles, quando no trabalho em grupo, expor suas opiniões.

Tabela 1 - Distribuição percentual dos discentes, de acordo com as respostas às questões fechadas do questionário ( $\mathrm{n}_{\text {graduação }}=30 / \mathrm{n}_{\text {pós-graduação }}=10$ ).

\begin{tabular}{lcccc}
\hline \multicolumn{1}{c}{ Questões } & \multicolumn{2}{c}{ Graduação } & \multicolumn{2}{c}{ Pós-graduação } \\
\hline $\begin{array}{l}\text { 1. Você já tinha vivenciado metodologias ativas } \\
\text { de ensino aprendizagem anteriormente? }\end{array}$ & 33 & 67 & 30 & 70 \\
$\begin{array}{l}\text { 2. Você acha válida a utilização de } \\
\text { metodologias ativas na graduação? }\end{array}$ & 100 & 0 & 100 & 0 \\
$\begin{array}{l}\text { 3. Você se sente à vontade para colocar suas } \\
\text { opiniões? }\end{array}$ & 96 & 4 & 100 & 0 \\
$\begin{array}{l}\text { 4. Você gostaria de conhecer mais sobre } \\
\text { metodologias ativas de ensino aprendizagem? }\end{array}$ & 87 & 13 & 100 & 0 \\
$\begin{array}{l}\text { 5. Você utilizaria esta metodologia futuramente } \\
\text { no seu exercício profissional? }\end{array}$ & $*$ & $*$ & 100 & 0 \\
\hline
\end{tabular}

* Questão não aplicada aos graduandos.

As falas mais significativas para ilustrar as principais opiniões emitidas estão apresentadas a seguir.

\section{Alunos da Graduacão}

Apenas dois alunos responderam já terem vivenciado experiências com metodologias ativas na graduação, sendo um deles em atividades extracurriculares:

"No PET" \{Programa de Educação

Tutorial\} (A12)

"Poucas vezes" (A22)

Quanto à questão sobre a validade do uso de metodologias ativas, algumas falas analisadas foram relacionadas ao pensamento crítico e à construção de competências de comunicação:

"O aluno é levado a pensar também e não só a escutar" (A1)

"Estimula o aprendizado e o senso crítico" (A8)
"Colocando a questão desta forma todos podem opinar e discutir" (A17) "As opiniões e conselhos dos colegas sempre acrescentam ideias sobre o assunto" (A23)

Dentre as justificativas para a facilidade de se comunicar, muitas falas destacaram a abertura proporcionada pelo trabalho em pequenos grupos:

"O grupo é pequeno e amigo" (A2)

"Muito à vontade e também para esclarecer minhas dúvidas" (A17)

"Abrem-se espaços para debates" (A25)

"Com o decorrer da aula as opiniões saem naturalmente" (A29)

As justificativas mais marcantes sobre a vontade de conhecer mais sobre metodologias ativas foram:

"Gostaria de conhecer os resultados positivos e negativos..." (A5) 
"Poderia ser utilizada em debates de casos clínicos" (A12)

Alunos de Pós-Graduação

Dos dez mestrandos, sete relataram nunca terem tido experiências com a metodologia. Entre as falas:

"Em minhas experiências como aluno, foi o primeiro contato com o método" (M3)

"Todas as aulas que participei foram bancárias, nenhuma dessa forma" (M6)

Todos ainda relataram ser positiva a utilização de metodologias ativas na graduação, sendo de destaque:

"Acho válido transmitir parte da responsabilidade de buscar o conhecimento para o aluno" (M3)

"Favorece o crescimento do aluno para aprendizado independente, pesquisa, comunicação, e consolidação e memorização do conteúdo discutido" (M6)

Quanto à liberdade em expressar opiniões, os mestrandos disseram:

"Nesse tipo de metodologia acho que todas as ideias são válidas" (M1)

"De uma maneira mais informal nos sentimos mais a vontade para dizer o que pensamos sobre os assuntos" (M10)

Todos também relataram querer conhecer mais sobre metodologias ativas, justificando:

"Acho que as metodologias ativas têm muito espaço para se desenvolver no ensino odontológico" (M4)

"Sim, como aluno de mestrado, interesso-me por metodologias de ensino" (M9)

Na questão 5, respondida apenas pelos mestrandos, sobre a intenção de uso de metodologias ativas no futuro profissional, as respostas mostraram uma percepção positiva e interessante:

"Ela é muito válida, pois faz o aluno buscar aquilo que ele tem necessidade de aprender e saber" (M2)

"Seria uma ferramenta a ser utilizada para estimular o aluno a procurar" (M6)

"Para alguns assuntos" (M8)

\section{DISCUSSÃO}

As DCN do Curso de Graduação em Odontologia preconizam o perfil do Cirurgião-Dentista baseado em uma formação generalista, humanista, crítica e reflexiva, para atuar em todos os níveis de atenção à saúde, com base no rigor técnico e científico. Além disso, o profissional deve estar capacitado ao exercício de atividades referentes à saúde bucal da população, pautado em princípios éticos, legais e na compreensão da realidade social, cultural e econômica do seu meio, dirigindo sua atuação para a transformação da realidade em benefício da sociedade ${ }^{22}$. Para este nível de formação, fica clara a necessidade de modelos pedagógicos que possibilitem a construção ativa do conhecimento, que deverá prolongarse por toda a vida profissional. A integração ensino-serviço-comunidade e a utilização de metodologias ativas de ensino-aprendizagem são apontadas como estratégias para a formação de profissionais voltados para o mundo do trabalho e para as necessidades da população, visando o aprendizado significativo $^{18,22}$.

Quanto aos resultados, pode-se observar que a maioria dos alunos de graduação não estava familiarizada com esse tipo de metodologia. Isso se deve a metodologia de ensino empregada na grande maioria das escolas de ensinos fundamental e médio brasileiros, que é tradicional $^{18}$. 
Entretanto, os entrevistados demonstraram satisfação com o método de ensino, o que desmistifica a ideia da dificuldade de aceitação de novas metodologias pelos alunos observadas neste trabalho. Analisando as opiniões, pode-se perceber que os acadêmicos valorizam a oportunidade de poder compartilhar suas experiências e conhecimentos, além de se sentirem mais motivados em uma aula onde suas opiniões são ouvidas. Estes resultados estão de acordo com os encontrados por Pires e Bueno ${ }^{23}$, que avaliaram a aceitação de alunos de graduação quando estes participaram de um curso de capacitação do programa de ensino em Saúde da Família fora da universidade, que utilizava a problematização. Os resultados revelaram aceitação desta metodologia. Na Austrália, na Universidade de Adelaide e na Universidade de Sidney, a ABP também foi bem aceita pelos estudantes ${ }^{24,25}$.

Quanto aos acadêmicos de pósgraduação, as impressões sobre a metodologia ativa também foram positivas. A maioria demonstrou interesse e satisfação pela metodologia e relatou que gostaria de utilizála futuramente no exercício docente. Ressaltase aqui a importância do ensino e uso de metodologias ativas para os acadêmicos de pós-graduação strictu sensu. Como professores do futuro, é importante que estes profissionais estejam familiarizados com essas metodologias que contribuem para a consolidação de um ensino odontológico em consonância com as $\mathrm{DCN}^{2}$.

A necessidade de mudança no ensino das profissões de saúde tem sido sentida desde a década de 50, quando se originou na Case Western Reserve University, a Aprendizagem Baseada em Problemas (ABP). Desde então, diversas universidades, principalmente na América do Norte, adotaram estratégias semelhantes. Em 1960, a McMaster University do Canadá reformou seu currículo com base na ABP e tornou-se uma de suas principais vitrines para o mundo. No Brasil, algumas faculdades de Medicina adotaram a ABP como estrutura curricular ${ }^{26}$. A enfermagem é outro curso da área da saúde que explora as metodologias ativas, principalmente a problematização, para melhorar a aprendizagem de seus alunos ${ }^{27}$. Entretanto, a utilização dessas metodologias é explorada na Odontologia e existem relatos pontuais de experiências em disciplinas específicas $^{10-16,18,19}$, mas não existe no curso de Odontologia brasileiro que utilize a $\mathrm{ABP}$ como estrutura curricular, ou seja, todos os conhecimentos essenciais do currículo são trabalhados a partir de problemas de ensino. $\mathrm{O}$ ensino odontológico ainda está seguindo o padrão de aulas expositivas, com o conhecimento sendo explorado de forma compartimentalizada, centrado na figura do professor que, em sua grande maioria, ainda segue a ideologia baseada no conceito tecnicista $^{6,7}$. Por outro lado, o desenvolvimento de competências relacionadas à capacidade de comunicação, tomada de decisões e liderança, como destaca o Art. $4^{\circ}$ das $\mathrm{DCN}^{19}$, certamente é garantido no trabalho em pequenos grupos, onde os espaços para o crescimento individual são garantidos, respeitando-se o trabalho em equipe e a construção coletiva dos saberes e fazeres.

Algumas experiências no campo da Odontologia têm sido relatadas fora do Brasil. Efetivamente tomam força, somente a partir de 1995 e provavelmente, algumas das primeiras escolas de Odontologia ${ }^{28}$ a adotarem a APB foram a Universidade de Adelaide, em 1993, e a de Harvard ${ }^{29}$, em 1995. Ou seja, a história da ABP nos cursos 
de Odontologia é relativamente curta, com menos de 20 anos de experiência. Vários países já aderiram a $\mathrm{ABP}^{30}$ : Canadá, Irlanda, Suécia, Inglaterra, Tailândia. Fincham et al. ${ }^{31}$ relataram que a $\mathrm{ABP}$ leva os estudantes a aprenderem tão bem quanto - se não mais que - o ensino tradicional e acredita-se que com esta abordagem os mesmos estejam melhor preparados para enfrentar o mundo em constante mudança do século XXI. Outro local que tem larga experiência com $\mathrm{ABP}$ em Odontologia é a Austrália ${ }^{32}$, onde todas as escolas de Odontologia a implantaram na década de 90.

Algumas experiências sobre o uso de metodologias ativas no Brasil na área da odontologia foram relatadas ${ }^{10-16,18,19}$. As metodologias ativas fundamentam-se em métodos de aprimorar o processo de aprender, com utilização de experiências reais ou inventadas, objetivando resolver, com sucesso, desafios advindos das atividades da profissão, em diferentes contextos ${ }^{25}$. Além disso, estimulam e desenvolvem nos alunos atitudes críticas e criativas em relação ao meio em que vivem e à profissão para a qual se preparam. A ABP possibilita a integração da teoria com a prática e também desenvolve o raciocínio lógico, a comunicação e a aprendizagem auto-dirigida ${ }^{29}$. Portanto é uma ótima alternativa pedagógica a ser utilizada na formação profissional.

Existem algumas pesquisas mostrando a resistência de muitos professores da área da saúde em romper com a metodologia tradicional, sendo que a principal dificuldade é a falta de um treinamento específico para a formação de tutores nessa nova metodologia $2,6,7, \mathrm{O}$ curso de Odontologia da UEM, a partir das ações propostas no projeto Pró-Saúde, vem realizando oficinas de capacitação em metodologias ativas de ensino-aprendizagem para seus docentes com as demais áreas da saúde, além do tema ser pauta das discussões em reuniões pedagógicas do curso. Acredita-se que o aprofundamento do estudo dessas metodologias, bem como sua disseminação, possa contribuir com os docentes e o curso para vencer as dificuldades e melhorar a qualidade do ensino praticado em nosso país. Desta maneira, é importante a capacitação permanente dos educadores fundamentada na DCN, que visam formar um profissional crítico e reflexivo que possa atuar com o perfil necessário às necessidades dos serviços de saúde e da população ${ }^{2,4,5}$.

Vale ressaltar que este estudo é um relato de experiência de um grupo de alunos de graduação e pós-graduação em uma única instituição. Além disso, há algumas limitações metodológicas que devem ser reportadas como a dificuldade na amostragem, validação do questionário e análise dos dados. Assim faz-se necessário novas pesquisas em outras IES e com uma amostra maior de discentes para confirmação destes achados. Futuros estudos longitudinais sobre a implementação de novas metodologias no curso poderão contribuir para a tomada de decisões sobre o melhor caminho na busca de uma formação de excelência.

\section{CONSIDERAÇÕES FINAIS}

Mudanças envolvem pessoas, valores, culturas e, especificamente no campo da saúde e da educação, envolvem também questões ideológicas, sociais, econômicas e históricas. Isso significa romper com antigos paradigmas, sem negar, entretanto, a historicidade das profissões, o acúmulo de conhecimentos e os modelos de atenção à saúde existentes no país. Neste contexto, as metodologias ativas são uma alternativa metodológica com potencial didático- 
pedagógico para preparar o futuro profissional e cidadão, necessária para ajudar a enfrentar os desafios de uma sociedade globalizada que passa por rápidas transformações. A partir dos resultados deste trabalho, pode-se sugerir uma tendência a aceitação e satisfação com metodologias ativas de ensino-aprendizagem, tanto pelos acadêmicos de graduação como os de pós-graduação.

\section{ABSTRACT \\ Acceptance of the use of active methodologies during training sessions in the SUS by undergraduate and graduate Dental students}

The aim of the study was to evaluate the acceptance of the use of active teachinglearning methodologies during training sessions in the SUS (Brazilian National Health System) with quantitative and qualitative data analyses. Thirty undergraduate and 10 graduate students answered a questionnaire after their participation in activities using the methodology. The data showed that $67 \%$ of undergraduate and $70 \%$ of graduate students had never experienced active teachinglearning methodologies. Moreover, the students showed great satisfaction with the new method that was totally approved. The active methodologies were shown to be a feasible didactic and pedagogical alternative with acceptance among undergraduate and graduate students, in accordance with the National Guidelines for Dentistry Course Curricula in Brazil.

Descriptors: Dentistry. Problem-Based Learning. Higher Education.

\section{REFERÊNCIAS}

1. Giffoni MRM. Docência em saúde: temas e experiências. Interface. 2007; 11(21):173-6. Acesso em 29 fevereiro 2016. Disponível em: http://www.scielo.br/scielo.php?script= $\underline{\text { sci_arttext\&pid=S1414-328320070001 }}$ $\underline{00018 \& \operatorname{lng}=\mathrm{pt} .}$

2. Lazzarin HC, Nakama L, Cordoni JL. O papel do professor na percepção dos alunos de odontologia. Saude Soc. 2007; 16(1):90-101. Acesso em 29 fevereiro 2016. Disponível em: http://www.scielo.br/scielo.php?script= sci_arttext\&pid=S0104-129020070001 $00009 \& \operatorname{lng}=\mathrm{pt}$.

3. Toassi RFC, Souza JM, Baumgarten A, Rösing CK. Avaliação curricular na educação superior em odontologia: discutindo as mudanças curriculares na formação em saúde no Brasil. Rev ABENO. 2012; 12(2):170-7. Acesso em 29 fevereiro 2016. Disponível em: http://revabeno.emnuvens.com.br/revabeno /article/view/120.

4. Fonseca EP. As diretrizes curriculares nacionais e a formação do cirurgiãodentista brasileiro. J Manag Prim Health Care 2012; 3(2):158-178. Acesso em 29 fevereiro 2016. Disponível em: http://www.jmphc.com/ojs/index.php/01/ar ticle/viewArticle/58.

5. Noronha DVC, Araújo SGC, Guedes EGB, Ferreira LJJ, Alencar SA. Teaching-learning process in times of the Unified Health System (SUS): training of faculty and dental surgeons in Brazil. Rev Odonto Ciênc 2010; 25(1):92-96. Acesso em 29 fevereiro 2016. Disponível em: http://www. scielo.br/scielo.php?script=sci_arttext $\underline{\text { \&pid }=\text { S1980-65232010000100019\& }}$ $\underline{\operatorname{lng}=\mathrm{pt}}$.

6. de Deus JM, Alves DRN, Figueiredo RR, Silva MMM, Amaral AF, Bollelal VR. Aula centrada no aluno versus aula centrada no professor: Desafios para a mudança. Rev Bras Educ Med. 2014, 
38(4):419-426. Acesso em 29 fevereiro 2016. Disponível em: http://www. scielo.br/pdf/rbem/v38n4/02.pdf.

7. Noro LR, Farias-Santos BCS, Sette-deSouza PH, Cruz RKS, Pinheiro IAG, Borges REA, et al. O professor (ainda) no centro do processo ensino aprendizagem em Odontologia. Rev ABENO. 2015; 15(1):2-11. Acesso em 29 fevereiro 2016. Disponível em: http://revabeno.emnuvens.com.br/revab eno/article/view/146.

8. Berbel NAN. As metodologias ativas e a promoção da autonomia de estudantes. Semina: Ciênc Soc Hum. 2011; 32(1):25-40. Acesso em 29 fevereiro 2016. Disponível em: http://www. proiac.uff.br/sites/default/files/docume ntos/berbel_2011.pdf.

9. Freitas VP, Carvalho RB, Gomes MJ, Figueiredo MC, Faustino-Silva DD. Mudança no processo ensino aprendizagem nos cursos de graduação em odontologia com utilização de metodologias ativas de ensino e aprendizagem. RFO. 2009; 14(2):163167. Acesso em 29 fevereiro 2016. Disponível em: http://www.upf.br/seer/ index.php/rfo/article/view/707.

10. Marcondes FK. Experiências no uso de metodologias ativas no ensino de Fisiologia, em um curso de graduação em Odontologia. III Simpósio Internacional de Inovação em Educação 2015; 1-10. Acesso em 29 fevereiro 2016. Disponível em: http://www. lantec.fe.unicamp.br/inova2015/images /trabalhos/artigos/T4.pdf.

11. Santos FA. Atualização das várias experiências e metodologias ativas em aplicação no Brasil. In XII Encontro Nacional de Dirigentes de Graduação;
2015. Acesso em 29 fevereiro 2016. Disponível em: http://eventos.funadesp. org.br/xii_encontro_graduacao/docs/ap resentacao/fabio_andre santos.pdf.

12. Moreira TP, Noro LRA, Diniz RCM, Antunes DK, Brasil CCP, Veras FMF, et al. Metodologia da problematização: uma escolha político-pedagógica. In: Noro, Luiz Roberto Augusto (Org.). Curso de Odontologia da Unifor: 10 anos ensinando e aprendendo. Fortaleza: Universidade de Fortaleza, 2005. Acesso em 29 fevereiro 2016. Disponível em: http://hp.unifor.br/ pdfs_notitia/880.pdf.

13. Toassi RFC, Stobäus CD, Mosquera JJM, Moysés SJ. Currículo integrado no ensino de Odontologia: novos sentidos para a formação na área da saúde. Interface. 2012; 16(41):529-42. Acesso em 29 fevereiro 2016. Disponível em: http://www.scielo.br/pdf/icse/v16n41/a op1712.

14. Borges RN, Melo M, Barcelos BA, Freitas GC. Utilização da metodologia "problem based learning" na disciplina de oclusão, na FO/UFG. Rev Odontol Bras Central. 2015; 24(71). Acesso em 29 fevereiro 2016. Disponível em: http://www.robrac.org.br/seer/index.ph p/ROBRAC/article/view/767/826.

15. Vaz SLA, Maia RMLC, Veloso TRG, Barros LAP. Análise transversal do aprendizado baseado em problemas na disciplina de estomatologia. Rev Brasil Pesq Saúde. 2012; 14(2):5-11. Acesso em 29 fevereiro 2016. Disponível em: http://periodicos.ufes.br/RBPS/article/v iewFile/4180/3304.

16. Oliveira RG, Dias AL, Júnior AMLF, Porto FR, Hespanhol FL, Silva RHA, Ricardo DR. Problematização como 
método ativo de ensino-aprendizagem em um Curso de Odontologia. Rev ABENO. 2015; 15(2):74-81. Acesso em 29 fevereiro 2016. Disponível em: http://revabeno.emnuvens.com.br/revab eno/article/view/180.

17. Araujo A, Slomski V. Active Learning Methods - An Analysis of Applications and Experiences in Brazilian Accounting Teaching. Creative Educ 2013; 4:20-27. Acesso em 29 fevereiro 2016. Disponível em: http://www. scirp.org/journal/PaperInformation.asp $\mathrm{x}$ ?PaperID=41425.

18. Matias KK. Metodologias de ensino e práticas pedagógicas em um curso de graduação em odontologia. Tese (Doutorado); Universidade Federal de Goiás, 2013. Acesso em 29 fevereiro 2016. Disponível em: https://reposi torio.bc.ufg.br/tede/bitstream/tede/3734 15/Tese\%20-\%20Karolina\%20Kellen\% 20Matias\%20-\%202013.pdf.

19. Maciel JAC, Norte AEO, Feitosa SG, Moreira TP, Farias MR, Teixeira AKM. Percepção dos acadêmicos do curso de Odontologia sobre o uso do portfólio como método avaliativo. Rev Eletr Gestão Saúde 2014; 5(2):279-289. Acesso em 29 fevereiro 2016. Disponível em: http://gestaoesaude.unb. br/index.php/gestaoesaude/article/view/ 737.

20. Almeida M (org). Diretrizes Curriculares Nacionais para os Cursos Universitários da Área de Saúde. $2^{a}$ ed. Londrina: Ed. Rede Unida; 2005.

21. Terada RSS, Hayacibara MF, Rigolon CJ, Silva MC, Lolli LF, Hidalgo MM. Implementação do Pró-Saúde no Curso de Odontologia da Universidade Estadual de Maringá. Rev ABENO.
2010; 10(2):64-71. Acesso em 29 fevereiro 2016. Disponível em: http://revabeno.emnuvens.com.br/revab eno/article/view/23.

22. Saliba NM, Moimaz SAS, Chiaratto RA, Tiano AVP. A utilização da metodologia PBL em Odontologia: descortinando novas possibilidades ao processo ensino-aprendizagem. Rev Odonto Ciênc 2008; 23(4):392-396. Acesso em 29 fevereiro 2016. Disponível em: http://www.maiza. com.br/adm/docencia/86.pdf.

23. Pires ROM, Bueno SMV. A problematização como modelo para ensino universitário: uma experiência piloto com alunos do $3^{\circ}$ e $4^{\circ}$ anos do curso de odontologia. Rev ABENO. 2006; 1(6):54-60. Acesso em 29 fevereiro 2016. Disponível em: http://abeno.org.br/ckfinder/userfiles/fil es/revista-abeno-2006-1.pdf.

24. Wetherell J, Mullins G, Hirsch R. Selfassessment in a problem-based learning curriculum in dentistry. Europ J Dent Educ. 1999; 3(3):97-105. Acesso em 29 fevereiro 2016. Disponível em: http://onlinelibrary.wiley.com/doi/10.1 111/j.1600-0579.1999.tb00074.x/epdf.

25. Gerzina TM et al. Student use and perceptions of different learning aids in a Problem-Based Learning (PBL) Dentistry Course. J Dent Educ. 2003; 67(6):641-53. Acesso em 29 fevereiro 2016. Disponível em: http://www.jdent aled.org/content/67/6/641.long.

26. Yasbeck DCM, Azevedo LL, Siqueira MRL, Menezes VM. Novos rumos para a educação médica. Rev Brasil Educ Med. 2000; 2(24):26-30. Acesso em 29 fevereiro 2016. Disponível em: http://educacaomedica.org.br/UserFiles/Fil 
e/2000/Volume24-2/novos rumos.pdf.

27. Reibnitz KS, Prado ML. Processo de trabalho, processo educativo e formação em Enfermagem. In: Reibnitz KS, Prado ML. Inovação e Educação em Enfermagem. Florianópolis: Ed. Cidade Futura 2006; 79-108.

28. Grenwood F, Mullins G, Townsend G, Wetherell J, Winning T. Evaluation of the performance of graduates from a PBL dental course. In Anais da HERDSA Annual International Conference; 1999; Melbourne; Australian. Melbourne: Higher Education Research and Development Society of Australasia 1999; 1-13. Acesso em 29 fevereiro 2016. Disponível em: http://www.herdsa.org. au/branches/vic/Cornerstones/pdf/green woo.pdf.

29. Thammasitboon K, Sukotjo C, Howell H, Karimbux N. Problem-based learning at the Harvard School of Dental Medicine: self-assessment of performance in postdoctoral training. $\mathrm{J}$ Dent Educ 2007; 71(8):1080-9. Acesso em 29 fevereiro 2016. Disponível em: http://www.jdentaled.org/content/71/8/ 1080.full.pdf+html.
30. Rich SK, Kelim RG, Shuller CF. Problem-based learning versus a traditional educational methodology: a comparison of preclinical and clinical periodontics performance. J Dental Educ 2005; 69(6):649-6. Acesso em 29 fevereiro 2016. Disponível em: http://www.jdentaled.org/content/69/6/ 649.full.pdf+html.

31. Fincham AG, Baehner R, Chai $Y$, Crowe DL, Fincham C, Iskander M et al. Problem-based learning at the University of Southern California School of Dentistry. J Dent Educ 1997; 61(5):417-25.

32. Winning T, Towsend G. Problembased learning in dental education: what's the evidence for and against and is it worth the effort? Aust Dent J. 2007; 5(1):2-9. Acesso em 29 fevereiro 2016. Disponível em: http://onlinelibrary. wiley.com/doi/10.1111/j.1834-7819.20 07.tb00458.x/epdf.

Correspondência para:

Mitsue Fujimaki

e-mail:mfujimaki@uem.br

Departmento de Odontologia, Universidade Estadual de Maringá

Av. Mandacaru, 1550

87080-000 Maringá, Paraná 